BNL 40359

\title{
The approach to equilibrium in a quark-gluon plasma
}

\author{
Ulrich Heinz \\ Brookhaven National Laboratory \\ Upton, NY 11973 \\ U S A
}

BNL -40359

DE88 002117

\begin{abstract}
I review work done during the last year on the problem of color equilibration in a quark-gluon plasma.
\end{abstract}

\author{
Invited Talk \\ Sixth International Conference on \\ Ultrarelativistic Nuclear Collisions \\ QUARK MATTER 1987 \\ Schloss Nordkirchen, West Germany \\ August 24-28, 1987
}

\section{DISCLAIMER}

\begin{abstract}
This report was prepared as an account of work sponsored by an agency of the United States Government. Neither the United States Government nor any agency thereof, nor any of their employees, makes any warranty, express or implied, or assumes any legal liability or responsibility for the accuracy, completeness, or usefulness of any information, apparatus, product, or process disclosed, or represents that its use would not infringe privately owned rights. Reference herein to any specific commercial product, process, or service by trade name, trademark. manufacturer, or otherwise does not necessarily constitute or imply its endorsement, recommendation, or favoring by the United States Government or any agency thereof. The views and opinions of authors expressed herein do not necessarily state or reflect those of the United States Government or any agency thereor.
\end{abstract}

This manuscript has been authored under contract number DE-AC02-76CH00016 with the U.S. Department of Energy. Accordingly, the U.S. Government retains a non-exclusive, royalty-free license to publish or reproduce the published form of this contribution, or allow others to do so, for U.S. Government purposes. 


\section{THE APPROACH TO EQUILIBRIUM IN A QUARK-GLUON PLASMA}

I review work done during the last year on the problem of color equilibration in a quark-gluon plasma.

\section{Introduction}

A general review of the pre-equilibrium stage of quark-gluon plasma formation was given at the Quark Matter ' 86 meeting in Asilomar [1]. In this contribution, I will review recent work and new developments in this field that occurred during the last year.

The basic questions to be addressed in this paper are: How does the quark-gluon plasme, once formed in heavy-ion collisions, approach equilibrium? What are the basic equilibration time scales - how do they compare with the plasma lifetime before hadronization and freeze-out set in? In particular, how do the strong color anisotropies, which are presumably present in the initial formation stage (where color strings are supposed to be abundantly generated and subsequently decay through particle production), disappear and how fast, if at all, do the color degrees of freedom attain local equilibrium?

Knowledge of the equilibration time scale is very important, since it not only gives us an intuitive feeling for the reliability of the local equilibrium assumption underlying so far most dynamical pictures (in particular the hydrodynamic approach), but also enters formally into the calculation of transport coefficients $[2,3]$ and the non-equilibrium corrections to hydrodynamic behavior, as well as determines the damping of collective modes in the plasma $[3,4]$ which might have phenomenological consequences [5].

The approach that I wish to present here involves the following chain of arguments: (i) equilibration is related to dissipation of energy and creation of entropy; (ii) it is determined by the dissipative, i.e., imaginary part of certain response functions - for example, to calculate the rate of dissipation for a density perturbation in an equilibrium plasma we need to work out the imaginary part of the retarded density-density correlation function; (iii) this imaginary part of the response function is dominated by its poles which signal collective modes - for example, phonons in the density-density correlation function, (colored) plasmons in the electric and magnetic response functions, etc.; (iv) from the imaginary part one can calculate a damping rate $\gamma$ for these collective modes which in turn yields an estimate for the equilibration time scale $\tau_{\text {equ }} \sim \hbar / \gamma$.

In this contribution I will concentrate on color equilibration and thus focus on color electric and magnetic plasma oscillations and their damping rates.

\section{Previous work}

In what I will call the "historical approach" (this includes the attempts reviewed in my QM ' 86 talk [1] ), one started from the retarded gluon propagator in a medium. This is the retarded correlation function for two color vector potentials $A_{\mu}$, describing in some 
sense the response of the system to an external color current [3a], but it is, of course, not gauge invariant. The naive hope in the "historical approach" was that, although the gluon propagator is not gauge invariant, maybe its poles are. Circumstantial evidence for this existed in the form of identical results in a variety of gauges for the real part of the pole position in the high-temperature, small-momentum limit (i.e., the plasma frequency and the plasmon dispersion relation at long wave lengths). However, for the imaginary part of the pole position, as well as for non-leading corrections in the high- $T$ expansion for the real part, this hope was not borne out by the calculations. Worse, it was found [6] that in some gauges (e.g. Coulomb gauge) not even the leading term in the real part came out correctly.

More specifically, the problem arose the following way: the condition for the pole in the gluon propagator signalling the presence of a longitudinal collective mode is [6]

$$
\mathbf{k}^{2}+\Pi_{00}\left(k_{0}, \mathbf{k}\right)=0 \text {. }
$$

Since $\Pi_{00}$ is generally complex, for real $\mathbf{k}$ the solution will be a complex frequency of the form $k_{0}(k)=\omega(k)-i \gamma(k)$. In the limit $|\gamma| \ll \omega(2.1)$ then reduces to the two equations

$$
\begin{aligned}
\mathbf{k}^{2}+\operatorname{Re} \Pi_{00}(\omega, \mathbf{k}) & =0 ; \\
\gamma(k) & =\frac{\operatorname{Im} \Pi_{00}(\omega, k)}{\partial \operatorname{Re} \Pi_{00}(\omega, k) / \partial \omega} .
\end{aligned}
$$

By explicit calculation in the 1-loop approximation one finds the following high-temperature expansion for $k \ll k_{0} \ll T$ :

$$
\Pi_{00}\left(k_{0}, \mathbf{k}\right)=N g^{2} \frac{\mathbf{k}^{2}}{k_{0}^{2}}\left[\frac{T^{2}}{9}+(a+i b) k_{0} T+c \frac{k_{0}^{2} T}{k}+\cdots\right]+\mathcal{O}\left(\frac{\mathbf{k}^{4}}{k_{0}^{4}}\right) .
$$

The gauge dependence mentioned above shows up as a gauge dependence of the constants $a, b$, and $c$ in this expression. In many gauges (for example, temporal axial gauge (TAG) and general covariant gauge (CoG)) the soefficient $c$ vanishes, and upon insertion of (2.4) into $(2,3)$ one obtains

$$
\omega^{2}=\frac{N g^{2} T^{2}}{9}+\mathcal{O}\left(k^{2}\right)
$$

independent of which one of these gauges is chosen, as well as

$$
\gamma=\frac{N g^{2}}{2} b T+\mathcal{O}(k)
$$

where $b$, however, is different in TAG and CoG (indeed, it depends on the gauge parameter in general $\mathrm{CoG}$ [6] ). On the other hand, in Coulomb gauge (CG) $c$ does not vanish, and since for small $k$ the term $\sim c$ swamps the two other terms in (2.4) (which were responsible for the leading behavior in the other gauges), the small-momentum expansion is seen to break down completely. This is what you get for considering a gauge dependent quantity to begin with. 


\section{3. "Gauge covariant" linear response approach}

During the past year or so Th. Elze, K. Kajantie, and T. Toimela from Helsinki and myself [6-9] have attempted to overcome the problem of the rather complicated gauge transformation properties of $\Pi_{\mu \nu}$ by generalizing the linear response framework for color electric and magnetic response in such a way that we end up with equations that transform covariantly, i.e., as vectors under gauge transformations. Although this is still not a gauge invariant formulation, at least the gauge transformation properties are under tight control [9], and we have more reason to hope that we will be able to extract gauge invariant answers from such an approach.

We start from the general linear response formula describing the change in the expectation value of an observable $\mathcal{O}(x)$ induced by an external perturbation described by the Hamiltonian density $\mathcal{H}_{\text {ext }}\left(x^{\prime}\right)[10]$ :

$$
\delta\langle\mathcal{O}(x)\rangle=-\frac{i}{\hbar} \int d^{4} x^{\prime} \Theta\left(t-t^{\prime}\right) \operatorname{Tr}\left\{\rho_{0}\left[\hat{\mathcal{O}}(x), \hat{\mathcal{H}}_{\text {ext }}\left(x^{\prime}\right)\right]\right\} .
$$

$\rho_{0}$ is the density operator $\left(\sim e^{-\beta \hat{H}_{0}}\right)$ for the equilibrium system, and the trace Tr over physical states is best evaluated using the path integral formulation given in $[11,12]$.

To study the color electromagnetic response, we consider the choice

$$
\mathcal{H}_{\text {ext }}(x)=\mathbf{E}(x)_{a} \cdot \mathcal{E}_{a}(x)-\mathbf{B}_{a}(x) \cdot \mathcal{B}_{a}(x) .
$$

In QED this is, up to a total derivative term, equivalent to the coupling $\mathcal{H}_{\text {ext }}=\mathcal{J}_{\mu}(x) A^{\mu}(x)$ with an external current $\mathcal{J}_{\mu}(x)$. In QCD this is no longer true, but the coupling (3.2) has the advantage of being gauge invariant under simultaneous gauge transformations of the quantum field $\mathrm{E}, \mathrm{B}$ and the sources $\mathcal{E}, \mathcal{B}$, while the $\mathcal{J}_{\mu} A^{\mu}$ coupling is not [12]. Note that in (3.2), to insure that one calculates the response to a physically identical electric or magnetic source when going from one gauge to another, the source has to be transformed by the gauge transformation connecting the two gauges: $\mathcal{E}(x) \rightarrow U(x)$ $\mathcal{E}(x) U^{-1}(x)$, etc.

I will focus on color electric oscillations generated by a pulse $\mathcal{E}_{i}(x)=-\delta(t) \partial_{i} \exp (i \mathbf{k} \cdot \mathbf{x})$ [6] for which, at least in the long wavelength limit, the magnetic part of $\mathcal{H}_{\text {ext }}$ can be neglected [8]. The response to a static magnetic field has been studied in [8], and a source corresponding to an electromagnetic plane wave is considered in present work by Mrówczyński [13].

The induced electric field in the plasma is given through (3.1) as

$$
\delta\left(E_{i}^{a}(x)\right)=-\frac{i}{\hbar} \int d^{4} x^{\prime} \theta\left(t-t^{\prime}\right) \operatorname{Tr}\left\{\rho_{0}\left[\hat{E}_{i}^{a}(x), \hat{E}_{j}^{b}\left(x^{\prime}\right)\right]\right\} \mathcal{E}_{j}^{b}\left(x^{\prime}\right),
$$

i.e., the electric response function is given in terms of a retarded cornmentator of two electric field operators:

$$
R_{i j}^{a b}\left(x-x^{\prime}\right)=-\frac{i}{\hbar} \theta\left(t-t^{\prime}\right) \operatorname{Tr}\left\{\rho_{0}\left[E_{i}^{a}(x), E_{j}^{b}\left(x^{\prime}\right)\right]\right\} .
$$


Obviousiy, $R_{i j}^{a b}$ is not a gauge-invariant quantity. Eq. (3.3), after being contracted with the $\mathrm{SU}(3)$ generators $\lambda_{a}$, transforms as a vector equation under gauge transformations, $U(x)$ [Eq. (3.3)] $U^{-1}(x)$; however, due to the folding over $x^{\prime}$ in Eq. (3.3), the response function (3.4) by itself still has a rather complicated gauge transformation behavior which has to be properly taken into account [9] when evaluating $R_{i j}^{a b}$ in different gauges.

The most convenient gauge to evaluate (3.4) [14] is the temporal axial gauge (TAG), $A_{0}^{a}=0$. In this gauge the Fock space spanned by $\left|A_{i}\right\rangle$ contains no dynamical unphysical (ghost) states, although the residual freedom of time-independent gauge transformations leads to $1 / p_{0}$ singularities in the propagators which have to be dealt with properly. (We will use the principal value prescription for these poles which was shown [15] to give correct results at 1 -loop order.) Therefore, up to this ambiguity in the static sector, the sources $\mathcal{E}$ and $\mathcal{B}$, expressed through the vector potential $\boldsymbol{A}$ and its derivatives, involve only physical degrees of freedom and are thus well-defined. Furthermore, the electric field is simply given by $E_{i}^{a}=-\partial_{t} A_{i}^{a}$ and does not involve the non-abelian structure constants. Another gauge, where only physical modes propagate, is the Coulomb gauge (CG), $\nabla \cdot \mathbf{A}=0$. In this gauge, however, $E_{i}^{a}=-\partial_{t} A_{i}^{a}+\partial_{i} A_{0}^{a}+g f_{a b c} A_{i}^{b} A_{0}^{c}$ involves also terms bilinear in the gluon potential, and the electric response function becomes more complicated [6]. In most other gauges further complications arise from propagating ghost states rendering the evaluation of (3.4) even more difficult [9].

\section{Temporal axial gauge}

Since in TAG, $\mathbf{E}$ is just the time derivative of $\mathbf{A}$, the response function (3.4) is easily expressed [16] in terms of the retarded gluon propagator alone:

$$
-i \theta\left(t-t^{\prime}\right) \operatorname{Tr}\left\{\left[E_{i}^{a}(x), E_{j}^{b}\left(x^{\prime}\right)\right]\right\}=-\delta^{(4)}\left(x-x^{\prime}\right)+\partial_{t} \partial_{t^{\prime}} D_{i j}^{a b R}\left(x-x^{\prime}\right) .
$$

In this gauge, therefore, all the information needed for the electric response function is actually contained in the gluon propagator. Its general structure in TAG (where $\mathcal{D}_{00}=$ $\mathcal{D}_{0 i}=0$ ) is given by

$$
\mathcal{D}_{i j}^{R}=\frac{1}{k^{2}-G}\left(\delta_{i j}-\hat{k}_{i} \hat{k}_{j}\right)+\frac{1}{k^{2}-F} \frac{k^{2}}{k_{0}^{2}} \hat{k}_{i} \hat{k}_{j},
$$

with

$$
\begin{aligned}
& G=\frac{1}{2}\left(\frac{k^{i} \Pi_{i j}^{R} k^{j}}{\mathbf{k}^{2}}-\Pi_{i i}^{R}\right) ; \\
& F=-\frac{k^{2}}{k_{0}^{2}} \frac{k^{i} \Pi_{i j}^{R} k^{j}}{\mathbf{k}^{2}}=-\frac{k^{2}}{\mathbf{k}^{2}} \Pi_{00}^{R} .
\end{aligned}
$$

The last identity in (4.3) is only true if $k^{\mu} \Pi_{\mu \nu}^{R}=0$, which is indeed the case in TAG at the 1-loop level. The longitudinal electric modes are thus given by the solution of

$$
k^{2}-F\left(k_{0}, \mathbf{k}\right)=0 \quad \Longleftrightarrow \mathbf{k}^{2}+\Pi_{00}^{R}\left(k_{0}, \mathbf{k}\right)=0
$$


whereas the transverse part of the response function has its pole at

$$
k^{2}-G\left(k_{0}, \mathbf{k}\right)=0
$$

\section{Coulomb gauge}

As already mentioned, in CG the electric response function no longer can be reduced just to derivatives of the gluon propagator; the retarded 3- and 4-point functions enter also, due to commutators involving those terms in the electric field which are bilinear in the gluon potentials. If in CG those terms were neglected and only the contribution to (3.4) involving the gluon propagator were taken into account, one would again obtain Eq. (4.4) for the longitudinal modes. However, while in a high-T expansion (2.4) of $\Pi_{00}$ in this equation the coefficient $b$ (which is responsible for damping) comes out identical to the TAG result, the coefficient $c$ in $C G$ does not vanish (in contrast to TAG), and destroys the leading small momentum behavior found in TAG. The crucial role of the 3and 4-point contributions to (3.4) in CG is the following [6]: while being completely real and thus not affecting the coefficient $b$ which governs damping (these corrections modify the contribution to the propagator involving a loop with one physical, transverse gluon and one unphysical, longitudinal gluon which, if cut, does not correspond to a physical scattering or decay process), they modify the real part of $\Pi_{00}$ such that the coefficient $c$ effectively vanishes. After adding the 2-, 3-, and 4-point contributions into an "effective $\Pi_{00}^{R}$ ", the condition for the longitudinal mode $\mathbf{k}^{2}+\Pi_{o 0, \text { eff }}^{R}\left(k_{0}, \mathbf{k}\right)=0$ yields the same result as in TAG, namely

$$
\begin{gathered}
\omega_{L}^{2}=\omega_{p}^{2}+\frac{3}{5} k^{2}+\ldots \quad \text { where } \omega_{p}^{2}=\frac{N g^{2} T^{2}}{9} \\
\gamma_{L}(k \rightarrow 0)=\frac{N g^{2} T}{24 \pi} .
\end{gathered}
$$

Further, one finds in both gauges, after similar cancellations of infrared divergent terms in CG by the 3- and 4-point contributions, that the transverse modes are given by

$$
\omega_{T}^{2}=\omega_{p}^{2}+\frac{6}{5} k^{2}+\ldots
$$

with the same damping rate $\gamma_{T}(k \rightarrow 0)=\gamma_{L}(k \rightarrow 0)$. For finite $k$ the damping rates $\gamma_{L}$ and $\gamma_{T}$ are different and worked out in Ref. 9 . 


\section{General covariant gauge}

The computation of (3.4) in CoG was recently carried out in [9]. There not only the 3and 4-point functions are important, but also one has to be careful to include the proper gauge transformation of the electric field operators and source, which is time-dependent and includes dynamical ghost fields. The calculation is lengthy, and I refer the interested reader to the original paper [9]. However, the result again is identical to TAG (for all gauge parameters), as it has to be by construction because it is explicitly ensured that the response to the physically identical external source is calculated. However, it is interesting to follow the technical steps in [9] and see the role played by the ghost fields in this gauge. If anything, one sees that covariant gauge appears to be a very inconvenient gauge for this problem.

\section{The connection to kinetic theory}

I will not give any details here of the actual computation of $R_{i j}$; the methods used to do the Matsubara frequency sums and integrals for the 1-loop terms are presented in detail in Ref. [6]. One important point to note is that the imaginary part of the response function (which is responsible for dissipation) arises from the poles of the propagators under the loop-integral; these lead to $\delta$-functions which put the loop particles on mass-shell, and hence can be interpreted as the matrix element for a physical decay or scattering process between the external perturbation and the thermal gluons in the heat bath. As an example, one finds for the imaginary part of the longitudinal response function $\left(\omega_{ \pm}=\frac{\omega \pm k}{2} ; k_{ \pm}=\frac{k \pm \omega}{2}\right)$ :

$$
\begin{aligned}
\operatorname{Im} F=-\frac{1}{16 \pi k} & \left\{\theta(\omega-k) \int_{\omega_{-}}^{\omega_{+}} d p\left|\mathcal{M}_{L \rightarrow \bar{q} q}\right|^{2}\left[\left(1-n_{F}\right)\left(1-n_{F}^{\prime}\right)-n_{F} n_{F}^{\prime}\right]\right. \\
& -2 \theta(k-\omega) \int_{k_{+}}^{\infty} d p\left|\mathcal{M}_{L+q \rightarrow q}\right|^{2}\left[n_{F}^{\prime \prime}\left(1-n_{F}\right)-n_{F}\left(1-n_{F}^{\prime \prime}\right)\right] \\
& +\theta(\omega-k) \int_{\omega-}^{\omega_{+}} d p\left|\mathcal{M}_{L \rightarrow g g}\right|^{2}\left[(1+n)\left(1+n^{\prime}\right)-n n^{\prime}\right] \\
& \left.-2 \theta(k-\omega) \int_{k_{+}}^{\infty} d p\left|\mathcal{M}_{L+g \rightarrow g}\right|^{2}\left[n^{\prime \prime}(1+n)-n\left(1+n^{\prime \prime}\right)\right]\right\} .
\end{aligned}
$$

Here we have included the (gauge-independent) quark-loop contribution, and $n, n_{F}$ are the boson and fermion distributions. $n, n^{\prime}$ and $n^{\prime \prime}$ indicate evaluation at momentum $p, p^{\prime}=\omega-p$, and $p^{\prime \prime}=p-\omega$, respectively. We see that each term splits into a loss and a gain term: in the first line the process is pair decay of the electric perturbation into a thermal $\bar{q} q$ pair, and its inverse; for the decay (loss term), the final $q, \bar{q}$ states are multiplied with Pauli-blocking factors $1-n_{F}$ and $1-n_{F}^{\prime} ;$ for the inverse process (gain term) the now ingoing $q, \bar{q}$ states are just multiplied with thermal distributions $n_{F}, n_{F}^{\prime}$. While $q \bar{q}-($ first 
line) and $g g$-pair decay (third line) only happen for timelike momenta of the external field $\left(\omega^{2}>k^{2}\right)$, at spacelike momenta $\left(\omega^{2}<k^{2}\right)$ scattering processes can occur: in the second line the external field gets absorbed by scattering a quark with initial momentum $p^{\prime \prime}$ to momentum $p$, and is regenerated by the inverse process; again all ingoing states have a factor $n_{F}$ and all outgoing quark states a factor $1-n_{F}$. The fourth line describes the analogous scattering process off thermal gluons, with Pauli-suppression $1-n F$ on the final states replaced by Bose-enhancement $1+n$. The relevant matrix elements are [6]

$$
\begin{aligned}
& \left|\mathcal{M}_{L \rightarrow \bar{q} q}\right|^{2}=\left|\mathcal{M}_{L+q \rightarrow q}\right|^{2}=4 N_{f} g^{2} p^{2} \sin ^{2}(\mathbf{k}, \mathbf{p}) ; \\
& \left|\mathcal{M}_{L \rightarrow g g}\right|^{2}=\left|\mathcal{M}_{L+g \rightarrow g}\right|^{2}=N_{c} g^{2} \frac{k^{2}}{2}\left(1+\cos ^{2}\left(\mathbf{p}, \mathbf{p}^{\prime}\right)\right)\left|1-\frac{p^{2}}{\omega^{2}-k^{2}} \sin ^{2}(\mathbf{k}, \mathbf{p})\right| .
\end{aligned}
$$

We see [16] that the finite-temperature field theoretical calculation results in a dissipative part of the response function which is intuitively interpreted in terms of physical scattering and decay processes and takes the form of a Boltzmann-Nordheim collision term in kinetic theory! Therefore we can also understand the equilibration rates and time-scales in the language of kinetic theory. Writing the dissipative part in the generic form

$$
\operatorname{Im} F \sim \Gamma_{\text {loss }}-\Gamma_{\text {gain }} \equiv \Gamma,
$$

we easily check from the form of (7.1) that always

hence

$$
\frac{\Gamma_{\text {loss }}}{\Gamma_{\text {gain }}}=e^{\beta \omega} ;
$$

$$
\frac{\Gamma_{\text {gain }}}{\Gamma_{\text {loss }}-\Gamma_{\text {gain }}}=\frac{1}{e^{\beta \omega}-1}=f_{\text {eq }}
$$

is the equilibrium distribution for the plasmon perturbation. If the initial electric perturbation has a phase-space distribution $f(x t ; \mathbf{k} \omega)$ not too far from equilibrium, then its time evolution will be given by the Boitzmann-Nordheim equation

$$
\begin{aligned}
\frac{d f}{d t} & =-\Gamma_{\text {loss }} f+\Gamma_{\text {gain }}(1+f) \\
& =-f\left(\Gamma_{\text {loss }}-\Gamma_{\text {gain }}\right)+\frac{\Gamma_{\text {gain }}}{\Gamma_{\text {loss }}-\Gamma_{\text {gain }}}\left(\Gamma_{\text {loss }}-\Gamma_{\text {gain }}\right) \\
& =-\Gamma\left(1-f_{\text {eq }}\right) .
\end{aligned}
$$

Its solution will behave like

$$
f=f_{\mathrm{eq}}+a e^{-\Gamma t} .
$$

Hence, $\Gamma=\Gamma_{\text {loss }}-\Gamma_{\text {gain }} \sim \operatorname{Im} F$ is the equilibration rate. The factor of proportionality in this last equation is not determined by the rather heuristic arguments of this section; but we know from the linear response approach of sections 2,3 that, at least for perturbations with the plasmon dispersion relation (2.1), it is given by $-1 /(\partial \operatorname{Re} F / \partial \omega)$ (see Eq. (2.3)). 
I will now give the complete result [6] for this equilibration rate $\Gamma=\gamma$ in the zeromomentum limit, including both quark and gluon contributions and not invoking a hightemperature expansion:

$$
\gamma(k \rightarrow 0)=\frac{g^{2} \omega}{96 \pi}\left[2 N_{f} \operatorname{th} \frac{\omega}{4 T}+N_{c} \operatorname{cth} \frac{\omega}{4 T}\right],
$$

which, when evaluated on the dispersion relation at $\omega=\omega_{p} \sim g T \ll T$, yields

$$
\gamma(k \rightarrow 0) \simeq \frac{N_{c} g^{2} T}{24 \pi}+\frac{N_{f}\left(2 N_{c}+N_{f}\right) g^{4} T}{3456 \pi}
$$

One sees that the quark contribution $\sim N_{f}$ is suppressed by 2 orders in $g$ ! The reason is that for $\omega \sim g T$ the loop integral is dominated by small momenta $\leqslant g T$ where the gluon Bose distribution $\sim 1 / p$ becomes very singular, whereas the quark distribution saturates at 1 . So, at this order of $g$, equilibration of color perturbations is completely dominated by gluonic scattering and decay processes!

An order of magnitude estimate for the associated time scales can be obtained by assuming a typics! plasma temperature of $T=200 \mathrm{MeV}$ and a strong coupling constant $\alpha_{a} \cong 1$. We then get (the first number is just the leading order result, the number in brackets includes the quark loop contribution in Eq. (7.9))

$$
\frac{\gamma}{\omega_{p}} \simeq 0.2 \quad(0.3)
$$

(i.e., the oscillations are damped after 3-5 periods) and

$$
\tau=\frac{\hbar}{\gamma} \simeq 2 \mathrm{fm} / \mathrm{c} \quad(1.2 \mathrm{fm} / \mathrm{c}),
$$

i.e., color equilibration occurs after $1-2 \mathrm{fm} / \mathrm{c}$.

\section{The effective action approach}

In the remainder of this survey I will briefly review some recent work by H. Hansson and 1. Zahed at Stony Brook, who used a completely different method to try to answer the same question. Unfortunately their result is quite different from the one quoted above, and in their calculation $\gamma$ even comes out with the opposite sign, indicating instability of the plasma. I will briefly outline their method, show that it also deals with a gaugedependent quantity too, and quote their answer. The lesson to be learned from this is that, although things looked nice at the end of section 7 above, the final word on the equilibration time scale is not said, and the problem cannot be considered settled before the recurring problems with gauge-dependence are decisively eliminated. 
Hansson and Zahed start from the path integral expression for the partition function of a gluonic system in an external background field $A_{\mu}$, subject to an external source $J_{\mu}$ [18]:

$$
\begin{aligned}
\tilde{Z}[J, A] & =e^{i \widetilde{W}[J, A]} \\
& =\int \mathcal{D} Q \Delta_{G}[A, Q] \exp \left\{i \int d^{4} x\left[\mathcal{L}(A+Q)-\frac{1}{2 \xi}\left(G_{a}(A, Q)\right)^{2}+J_{\mu}^{a} Q_{a}^{\mu}\right]\right\} .
\end{aligned}
$$

At finite temperature and for $J=A=0,-i \widetilde{W}$ is the grand canonical potential. $G_{a}$ is a gauge-fixing condition for the quantum gluon field $Q_{\mu}^{a}$, which can be chosen such that, while breaking gauge invariance of the integrand with respect to transformations of $Q$, invariance with respect to gauge transformation of the background field $A$ is preserved. An example of such a gauge is background Frenkel-Taylor gauge, given by

$$
G_{a}(A, Q)=\beta\left(\partial_{b} \delta^{a c}+g f^{a b c} A_{0}^{b}\right) Q_{0}^{c}+\alpha\left(\delta^{a c} \nabla-g f^{a b c} \mathbf{A}^{b}\right) \cdot \mathbf{Q}^{c} .
$$

For different values of $\alpha$ and $\beta$ this gauge in the limit $A \rightarrow 0$ interpolates [19] between Coulomb gauge $(\alpha=1, \beta=0, \xi \rightarrow 0)$, covariant gauge $(\alpha=\beta=1, \xi$ arbitrary), and static gauge $(\alpha=1, \beta=\infty, \xi \rightarrow 0)$. Hansson and Zahed chose for non-vanishing $A$ the case $\alpha=\beta=1$, i.e. background covariant gauge.

$\Delta_{G}[A, Q]$ in (8.1) is the Fadeev-Popov ghost determinant, and can in the usual way be reexpressed as an integral over a ghost Lagrangian,

$$
\Delta_{G}[A, Q]=\int \mathcal{D}_{\eta} D \bar{\eta} \exp \left\{-i \int d^{4} \boldsymbol{x} \bar{\eta}_{a} \mathcal{M}_{a b} \eta_{b}\right\}
$$

where for the gauge (8.2)

$$
\mathcal{M}_{a b}=\frac{\delta G_{a}}{\delta Q_{\mu}^{c}}\left[D_{\mu}(A+Q)\right]_{c b}=\left[\beta D_{0}(A) D_{0}(A+Q)-\alpha \mathbf{D}(A) \cdot \mathbf{D}(A+Q)\right]_{a b}
$$

$\left(D_{\mu}(a)\right.$ is the covariant derivative, $\left.D_{\mu}^{a b}(a)=\partial_{\mu} \delta^{a b}+g f^{a c b} a_{\mu}^{c}\right)$.

From (8.1) the effective action is obtained by a Legendre transformation

$$
\widetilde{\Gamma}[\widetilde{Q}, A]=\widetilde{W}[J, A]-\int d^{4} x J_{\mu}^{a} \widetilde{Q}_{a}^{\mu},
$$

where the "classical field" $\widetilde{Q}_{\mu}^{a}$ is defined through

$$
\widetilde{Q}_{a}^{\mu}=\frac{\delta \widetilde{W}}{\delta J_{\mu}^{a}}
$$

The "effective background field action" is then defined as the $\widetilde{Q} \rightarrow 0$ limit of this expression,

$$
\Gamma[A] \equiv \tilde{\Gamma}[\tilde{Q}=0, A],
$$

and is, by construction, invariant [18] under gauge transformations on the background field $A$. Hence, it can depend only on gauge invariant combinations of components of the 
background field strength tensor and its covariant derivatives and has to take the general form

$$
\Gamma[A]=\frac{1}{2} \int \frac{d^{4} k}{(2 \pi)^{4}}\left[\epsilon(k) \mathbf{E}_{a}(k) \cdot \mathbf{E}_{a}(-k)-\frac{1}{\mu(k)} \mathbf{B}_{a}(k) \cdot \mathbf{B}_{a}(-k)+\ldots\right],
$$

where the dots denote nonlinear and derivative terms (see Ref. 17).

On the other hand, an explicit calculation of $\Gamma[A]$ in the 1-loop approximation yields it in the form

$$
\begin{aligned}
\Gamma[A]=-\frac{1}{2} \int \frac{d^{4} k}{(2 \pi)^{4}}\{ & A_{\mu}^{a}(k)\left(k^{2} g^{\mu \nu}-k^{\mu} k^{\nu}\right) A_{\nu}^{a}(-k)+ \\
& \left.+A_{\mu}^{a}(k) \Pi_{a b}^{\mu \nu}(k) A_{\nu}^{b}(-k)+\ldots\right\}
\end{aligned}
$$

where, from gauge invariance of $\Gamma$, one is assured [17] that the omitted higher order terms in $A$ in (8.9) correctly combine to give the nonlinear terms in $\mathbf{E}^{2}$ and $\mathrm{B}^{2}$ in (8.8). $\Pi_{a b}^{\mu \nu}$ in (8.9) describes polarization of the background field $A$ by loops of the quantum field $Q ;$ it is thus different from the vacuum polarization tensor usually considered, where no vertices to the background field enter. In fact, gauge invariance of $\Gamma[A]$ guarantees that this background polarization tensor is always transverse,

$$
k_{\mu} \Pi^{\mu \nu}=0 \text {, }
$$

independent of the specific gauge choice $G_{a}$ in (8.2) for the quantum field.

By comparison of coefficients in (8.8) and (8.9) we find at 1-loop order for the dielectric function $\epsilon(k)$ and the diamagnetic function $\mu(k)$

$$
\begin{aligned}
& \epsilon(k)=1+\frac{\Pi_{00}(k)}{\mathbf{k}^{2}}, \\
& \frac{1}{\mu(k)}=1+\left(\frac{3 \omega^{2}}{\mathbf{k}^{2}} \Pi_{00}-\Pi_{i i}\right) / 2 \mathbf{k}^{2} .
\end{aligned}
$$

The next step in their calculation is to minimize $\Gamma[A]$ to obtain equations of motion for the "classical" or "mean-field" modes for the background field in the medium. They derive equations for a transverse electric and magnetic as well as for a longitudinal electric mode, and the latter reduces to

$$
\epsilon(k)=0 \quad \mathbf{k}^{2}+\Pi_{00}(k)=0 .
$$

This is the same equation as we had it in TAG (see (2.1)), but now with a completely different $\Pi_{00}$, namely the one for the background field in covariant background gauge!

The surprise (at least to me) came when Hansson and Zahed [17] evaluated Eq. (8.13) and found $\epsilon(k)$ to depend on the gauge parameter $\xi !$ Although much care was taken to ensure that $\Gamma[A]$ was invariant under gauge transformations of $A$, it still turned out 
to depend on the gauge choice for the quantum field $Q$. Somehow the fact that the $J_{\mu} Q^{\mu}$ coupling to the source $J$ in (8.1) is not gauge invariant has propagated through the Legendre transformation (8.5) into the limit $\widetilde{Q} \rightarrow 0$ in Eq. (8.7) [20] which apparently leads to different results for different gauge choices $G_{a}(A, Q)$.

Specifically, (8.13) has the solution (in the high $T$, small $k$ limit)

$$
\begin{gathered}
\omega_{L}^{2}=\omega_{p}^{2}+\frac{3}{5} k^{2}+\ldots, \\
\gamma_{L}(k \rightarrow 0)=\frac{N g^{2} T}{24 \pi}\left(-11-\frac{(1-\xi)^{2}}{4}\right)
\end{gathered}
$$

(the same damping rate comes out for the transverse modes). Not only is $y$ gaugeparameter dependent, it also has, for any value of $\xi$, the "wrong" sign, indicating instability of the mean-field modes!

The dominant negative contribution in (8.15), the -11 , actually is the same -11 leading to asymptotic freedom. The Lorentz covariant nature of the gauge forces a relationship between the sign of the $\beta$-function and the sign of $\operatorname{Im} \Pi_{00}$ at zero temperature that is not present in non-covariant gauges like TAG. The relation between the $T=0$ result and the finite- $T$ medium corrections is such [7] that this same -11 then dictates the leading high- $T$ behavior of the damping rate (8.15) above, in this particular gauge.

The problem is obviously that the dispersion relation (8.13) in the effective action approach again involves a gauge-dependent quantity, $\Pi_{\mu \nu}$. Indeed, if one chooses background Coulomb gauge ( $\alpha=1, \beta=0, \xi \rightarrow 0$ in (8.2)), then Eq. (8.13) remains formally unchanged, but involves $\Pi_{00}$ in Coulomb background gauge which turns out [21] to be the same as $\Pi_{00}$ in the usual Coulomb gauge, and we are back to the infrared problem mentioned at the end of section 2.

In reference 17 it is argued that a completely gauge-independent version of $\Gamma$ can be defined [20], and that it agrees with their calculation for $\xi=0$. This appears to single out $\xi=0$, the Landau background gauge, as the one physical gauge in the whole class of covariant background gauges; however, the argument was only proven at $T=0$, and it is not entirely clear that it still applies at finite temperature.

In any case, even for $\xi=0$ the rate $\gamma$ is negative, and this calculation implies plasma instability, whereas we had found plasma equilibration. So what is going on? Are both approaches answering the same question? What is the relationship between linear response and minimizing the effective action? Which is the correct, gauge-invariant answer for the color equilibration rate in a quark-gluon plasma?

It is clear at this stage that, whatever prejudices one might have for or against either one of the two approaches, a lot more work has to be done to clarify the issue. Both approaches can be criticized by arguing that they involve gauge-dependent quantities, and this state of affairs has to be changed. The result by Hansson and Zahed is particularly troublesome, because at second order in perturbation theory, $\mathcal{O}\left(g^{2}\right)$, one would not expect 
to see such an instability unless one were somehow starting from the wrong ground state. In that case our whole picture of the QGP would have to change.

\section{REFERENCES}

1. U. Heinz, Nucl. Phys. A461 (1987) 49c.

2. A. Hosoya and K. Kajantie, Nucl. Phys. B250 (1985) 666;

P. Danielewicz and M. Gyulassy, Phys. Rev. D31 (1985) 53;

S. Gavin, Nucl. Phys. $\mathbf{A 4 3 5}$ (1985) 826.

3. (a)U. Heinz, Ann. Phys. 168 (1986) 148;

(b)W. Czyz and W. Florkowski, Acta Phys. Pol. B17 (1986) 819;

(c)A. Dyrek and W. Florkowski, Phys. Rev.D (1987), in press.

4. K. Kajantie and T. Matsui, Phys. Lett. B164 (1985) 373;

A. Bialas and W. Czyz, Acta Phys. Pol. B17 (1986) 635;

A. Bialas, contribution to this conference.

5. U. Heinz, Ann. Phys. 161 (1985) 48;

A. Bialas and W. Czyz, Z. Plys. C28 (1985) 255.

6. U. Heinz, K. Kajantie and T. Toimela, Phys. Lett. B183 (1982)96;

U. Heinz, K. Kajantie and T. Toimela, Ann. Phys. 176 (1987) 218.

7. Th. Elze, U. Heinz, K. Kajantie and T. Toimela, Z. Phys. C, in press.

8. Th. Elze, K. Kajantie and T. Toimela, Z. Phys. C, in press.

9. Th. Elze, K. Kajantie and T. Toimela, in preparation.

10. A. L. Fetter and J. D. Walecka, Quantum Theory of Many-Particle Systems, McGrawHill, San Francisco, 1971.

11. C. W. Bernard, Phys. Rev. D9 (74) 3312;

D. Gross, R. Pisarski and L. Yafie, Rev. Mod. Phys. $\underline{53}$ (1981) 43.

12. P. Ramond, Field Theory - A Modern Primer, Benjamin Cummings, Reading (Massachusetts), 1981, pp. 269 ff..

13. S. Mrówczyński, private communication.

14. K. Kajantie and J. Kapusta, Ann. Phys. 160 (1985) 477.

15. H. Cheng, E.-C. Tsai, Phys. Rev. Lett. 57 (1986) 511.

16. H. A. Weldon, Phys. Rev. D28 (1983) 2007.

17. T. H. Hansson and I. Zahed, Phys. Rev. Lett. $\underline{58}$ (1987) 2397, and Nucl. Phys. $\underline{B 292}$ (1987) 725.

18. L. F. Abbott, Nucl. Phys. B185 (1981) 189.

19. J. Frenkel and J. C. Taylor, Nucl. Phys. B109 (1976) 439.

20. A. Rebhan, Nucl. Phys. B288 (1987) 832.

21. U. Heinz, unpublished. 